УДК 548.527

ISSN 1606-867X

DOI: https://doi.org/10.17308/kcmf.2019.21/2368

Поступила в редакцию 02.11.2019

Подписана в печать 15.12.2019

\title{
Модель роста нитевидных кристаллов, контролируемого гетерогенной химической реакцией, с учетом размерного эффекта
}

\author{
๔2019 О. Д. Козенков ${ }^{1 \rrbracket}$, Д. А. Жукалин ${ }^{2}$, И. О. Бакланов ${ }^{1}$, Л. Г. Косырева ${ }^{1}$, \\ А. Ю. Климов ${ }^{1}$, И. С. Обозный ${ }^{1}$ \\ ${ }^{1}$ Военный учебно-научный центр ВВС «Военно-воздушная академия \\ Uм. профессора Н.Е. Жуковского и Ю.А. Гагарина» \\ ул. Старых Большевиков, 54 «А», 394064 Воронеж, Российская Федерация \\ ${ }^{2}$ Воронежский государственный университет \\ Университетская площадь, д. 1, 394018 Воронеж, Российская Федерация
}

\begin{abstract}
Аннотация. Нитевидные кристаллы имеют важное практическое применение в машиностроении позволяя улучшать прочностные, эксплуатационные и физические характеристики конструкционных материалов при создании композитов армированных усами. Модель роста усов кремния, контролируемого химической реакцией на границе жидкостьгаз, дополнена учетом давления насыщенного пара кремния в расплаве на вершине кристалла. Для кристаллов достаточно малых размеров давление насыщенного пара кристаллизующегося вещества становится столь значительным, что рост кристалла прекращается. Модель предполагает диффузионную доставку исходных веществ и отвод продуктов реакции в тонкий приповерхностный слой газа у поверхности раздела жидкость-газ, где концентрации реагентов остаются постоянными. Концентрации реагентов в этом слое определяют скорость химической реакции выделения кристаллизующегося вещества. Учтён поток, возникающий за счет давления насыщенного пара кремния над расплавом, при условии, что испаряющиеся атомы практически полностью вступают во взаимодействие с реагентами в газовой фазе. Баланс диффузионных, химических потоков и потоков испарения позволяет найти скорость роста нитевидного кристалла в зависимости от его радиуса и технологических параметров процесса. Зависимость скорости роста нитевидного кристалла от его радиуса имеет максимум, а при достаточно малых поперечных размерах кристалла обращается в нуль. Получено выражение для радиуса кристалла, при котором скорость роста обращается в нуль. Рост кристалла прекращается при достаточно больших радиусах. Получено выражение, определяющее максимальный радиус кристалла, при котором рост становится невозможным. Результаты работы будут полезны при анализе роста нитевидных кристаллов различных веществ, который сопровождается химической реакцией. Модель, предложенная в работе, будет востребована для разработки технологических процессов массового производства кристаллов с целью получения композиционных материалов.
\end{abstract}

Ключевые слова: нитевидный кристалл, гетерогенная химическая реакция, диффузионный поток, поток химической реакции, поток испарения, минимальный радиус, максимальный радиус.

\section{ВВЕДЕНИЕ}

Нитевидные кристаллы (НК, усы) - монокристаллические, «идеально совершенные», квазиодномерные объекты с поперечными размерами от сотен микрометров до десятков нано-

Козенков Олег Дмитриевич, e-mail: kozenkov_w@mail.ru метров, обладающие рядом уникальных свойств [1-5]. Они находят широкое применение для изготовления чувствительных элементов датчиков различных физических величин [6], модифицирования поверхностей твердых тел [7], создания композиционных материалов [1], армированных усами. НК представляют также и науч-

Контент доступен под лицензией Creative Commons Attribution 4.0 License.

The content is available under Creative Commons Attribution 4.0 License. 
ный интерес как модельные объекты для изучения физико-химических процессов роста и формообразования монокристаллов по механизму пар-жидкость-кристалл (ПЖК) [1-4]. Практически идеальное совершенство НК представляет интерес при изучении их структуры, особенно для усов с гетеропереходами и химических соединений. Особый практический интерес к НК возникает в связи с перспективами их применения в интенсивно развивающихся нанотехнологиях $[4,7,8]$.

Наиболее перспективным представляется использование НК в материалах машиностроения для улучшения их эксплуатационных свойств. В частности, усы, армирующие композиционные материалы, радикально улучшают прочностные и эксплуатационные характеристики конструкционных материалов машиностроения. Кроме того, использование усов, растущих в форме углеродных нанотрубок, позволяет улучшать некоторые физические свойства материалов машиностроения, в частности, повышать их теплопроводность по сравнению с теплопроводностью чистых металлов.

НК получают при физическом осаждении [4] или в системах, в которых рост НК связан с протеканием гетерогенной химической реакции выделения кристаллизующегося вещества $[1,2$, 3]. Независимо от способа получения усы различных материалов растут по механизму ПЖК, а закономерности этого механизма содержат общие черты, характерные для различных способов получения кристаллов.

Модели роста НК, описанные в литературе, не многочисленны. Так модель Дитмара-Ноймана [2] связывает процесс роста с диффузией кристаллизующегося вещества по боковой поверхности кристалла. Модель Чернова-Гиваргизова [2] учитывает размерный эффект Гиббса-Томсона и позволяет определить кинетические коэффициенты кристаллизации. Модель адсорбционно-стимулированного роста наноразмерных НК, предложенная в работе [4], основана на анализе процессов зарождения на границе кристалл-расплав. При достаточно малых размерах происходит переход от полицентрического к моноцентрическому зарождению, с чем связаны, по мнению авторов, кинетические особенности роста усов. В моделях, представленных в литературе не рассматривается процесс массообмена между растущим кристаллом и газовой фазой, тем более не рассматривается химическая реакция, приводящая к росту НК, хотя известные экспериментальные данные указывают на важность этих процессов для анализа роста $\mathrm{HK}$ :

- химическая реакция на границе жидкостьгаз выделения вещества растущего кристалла идет на поверхности расплава, выход реакции на кристаллической боковой поверхности уса и в объеме газа за счет гомогенного взаимодействия компонентов газовой фазы мал [1-4];

- скорость роста уса определяется концентрацией вещества, составляющего кристалл в расплаве, с ростом которой она падает $[10,11]$, и при концентрации $100 \%$ равна скорости роста на твердой кристаллической поверхности;

- скорость роста уса определяется площадью поверхности расплава на его вершине, с ростом которой она увеличивается [3];

- скорость аксиального роста НК зависит от температуры [12, 13], состава газовой фазы [14], скорости движения газовой смеси [3];

- скорость, с которой растут усы, определяется их радиусом $R$. При $2.5-5 \mu \mathrm{m} \leqslant R \leqslant 50 \mu \mathrm{m}$ скорость роста имеет обратно пропорциональную зависимость от $R$ [15], а при $R<2.5 \mu \mathrm{m}$ (при очень высокой плотности расположения усов на подложке) корень квадратный из скорости обратно пропорционален квадрату радиуса НК [2, 4];

- скорость роста усов зависит от плотности их расположения на монокристаллической подложке, причем с увеличением плотности усов на подложке она падает [16];

- вокруг вершины растущего НК образуется шаровая зона «питания», размер которой связан с поперечным размером кристалла и уменьшается по мере уменьшением радиуса НK [3, 16]. Для НК с поперечным размером $25 \mu \mathrm{m}$ размер его зоны «питания» составляет величину $\sim 500 \mu \mathrm{m}$;

- при определенных соотношениях исходных компонентов газовой фазы происходит травление кристаллизующегося вещества через жидкий расплав на вершине уса с образованием «отрицательных кристаллов» [17];

- НК различных материалов всегда растут конусными. Величина и знак конусности (радиальная периодическая неустойчивость) определяется исходным составом компонентов в газовой фазе, природой и составом расплава на вершине уса, а также температурой процесса роста [18, 19].

В работе [9] представлена модель роста HK, лимитируемого химической реакцией на границе расплав-газ, которая дала качественное объяснение основных закономерностей процесса роста НК с поперечными размерами свыше $2 \mu \mathrm{m}$ 
и обеспечила удовлетворительное согласие с известными экспериментальными данными.

Однако в модели [9] не учтено давление насыщенного пара кристаллизующегося вещества, испаряющегося с поверхности расплава на вершине НК. Если для микроразмерных кристаллов это не существенно, то для субмикро- и наноразмерных кристаллов поток испарения, возникающий с поверхности расплава, необходимо учитывать.

Целью работы является учет потока испарения кристаллизующегося вещества с поверхности жидкой фазы на вершине кристалла в модели роста НК, контролируемого гетерогенной химической реакцией.

\section{РЕЗУЛЬТАТЫ И ИХ ОБСУЖДЕНИЕ}

В рамках модели [9] учтем давление насыщенного пара кристаллизующегося вещества, зависящее от поперечного размера кристалла. Модель [9] построена для конкретного случая роста НК кремния в открытой проточной системе $\mathrm{H}_{2}+\mathrm{SiCl}_{4}$ с горизонтальным расположением реактора. Поскольку основными компонентами газовой фазы по данным термодинамического анализа являются $\mathrm{H}_{2}, \mathrm{HCl}$, и $\mathrm{SiCl}_{2}$, то аксиальный рост уса происходит в результате протекания химической реакции, схему которой можно записать в виде:

$$
\mathrm{SiCl}_{2(\Gamma)}+\mathrm{H}_{2(\Gamma)} \longleftrightarrow 2 \mathrm{HCl}_{(\Gamma)}+\mathrm{Si}_{(\mathrm{K})} .
$$

На рис. 1 показана схема, иллюстрирующая модель роста НК. Каплю расплава на верши-

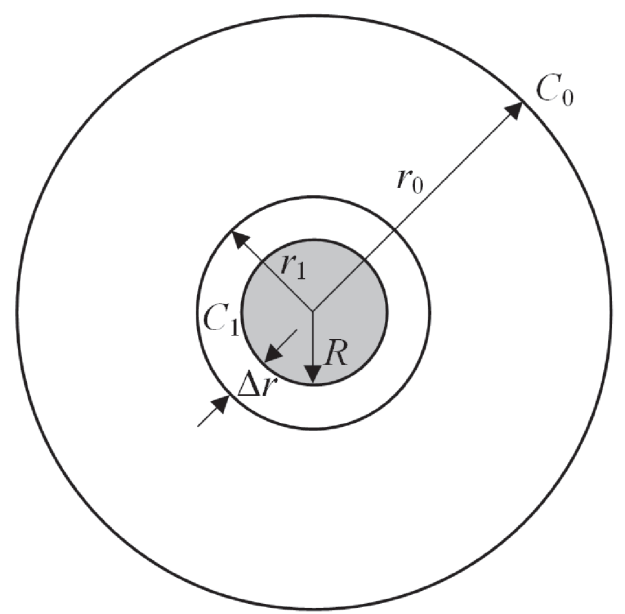

Рис. 1. Схема, иллюстрирующая модель роста НК контролируемого гетерогенной химической реакцией выделения кристаллизующегося вещества

[Fig. 1. Scheme illustrating the growth model of nanocrystals controlled by a heterogeneous chemical reaction for the release of a crystallizing substance] не НК заменили шаром радиуса $R$, площадь поверхности которого равна площади поверхности капли. В таком виде задача сводится к сферической симметрии: $r_{0}$ - радиус сферической поверхности, в пределах которой можно пренебречь движением газовой смеси в реакторе; $r_{1}$ - радиус сферической поверхности, в пределах которой концентрация компонентов газовой смеси $C_{1}$ остается постоянной в стационарном режиме роста; $C_{0}$ - концентрация основных компонентов исходной смеси в объеме газа; $\Delta r-$ толщина сферического слоя, в пределах которого концентрация компонентов газовой смеси остается постоянной для стационарного режима роста, (рис. 1).

Величина $\Delta r$ порядка длины свободного пробега молекул газовой фазы, что много меньше радиуса HK $R \gg \Delta r$. Из экспериментальных данных [3, 16], следует, что $R \gg r_{0}$.

Обозначим концентрации компонентов в объеме газовой фазы (за пределами сферической поверхности радиуса $\left.r_{0}\right) \mathrm{SiCl}_{2}, \mathrm{H}_{2}, \mathrm{HCl}$ как $C_{01}, C_{02}, C_{03}$ соответственно, а в приповерхностном слое на границе жикость-газ - $C_{1}, C_{2}, C_{3}$ соответственно.

Для стационарного роста НК диффузионные потоки компонентов $\mathrm{SiCl}_{2}, \mathrm{H}_{2}$, и $\mathrm{HCl}$ с учетом соотношений $R \gg \Delta r$ и $R \gg r_{0}$, запишем последнее в виде:

$$
\begin{aligned}
& J_{1}=4 \pi D_{1} R\left(C_{01}-C_{1}\right) ; \\
& J_{2}=4 \pi D_{2} R\left(C_{02}-C_{2}\right) ; \\
& J_{3}=4 \pi D_{3} R\left(C_{3}-C_{03}\right) .
\end{aligned}
$$

Здесь $J_{1}, J_{2}, J_{3}$ - диффузионные потоки основных компонентов исходной газовой смеси $\mathrm{SiCl}_{2}, \mathrm{H}_{2}$, и $\mathrm{HCl}$ соответственно; $D_{1}, D_{2}, D_{3}$ - коэффициенты диффузии для этих основных компонентов.

Как следует из стехиометрии схемы химической реакции (1), химический поток атомов кремния образуется за счет взаимодействия между равным числом молей исходных реагентов газовой фазы $\mathrm{SiCl}_{2}$ и $\mathrm{H}_{2}$, при этом число молей возникших атомов кремния равно числу молей исходных компонентов $\left(\mathrm{SiCl}_{2}, \mathrm{H}_{2}\right)$, которые приняли участие в реакции (1). Откуда следует, что протекание реакции (1) приводит к одинаковому уменьшению количества молей исходных компонентов газовой фазы системы $\left(\mathrm{SiCl}_{2}\right.$ и $\left.\mathrm{H}_{2}\right)$ и соответствующему увеличению в два раза количества молей продуктов реакции $(\mathrm{HCl})$.

$$
J_{1}=J_{2}=\frac{1}{2} J_{3} \text { или } J_{1}+J_{2}=J_{3}
$$


Обозначим $C_{01}-C_{1}=\Delta C_{1} ; C_{02}-C_{2}=\Delta C_{2}-$ убыль концентрации исходных компонентов газовой смеси $\mathrm{SiCl}_{2}$ и $\mathrm{H}_{2}$ в результате процесса диффузии от сферической поверхности радиуса $r_{0}$ до сферической поверхности радиуса $r_{1}$, а $C_{03}-C_{3}=-2 \Delta C_{3}-$ соответствующая убыль концентрации продукта реакции (1) $\mathrm{HCl}$ в результате диффузии в тех же границах, как показано на рис. 2.

Из выражений (2) и (3) получим:

$$
\Delta C_{2}=\Delta C_{1} \frac{D_{1}}{D_{2}}, \Delta C_{3}=2 \Delta C_{1} \frac{D_{1}}{D_{3}} .
$$

Будем считать реакционным объемом объем слоя толщиной $\Delta R$, как показано на рис. 2. Если учесть соотношение $R \gg \Delta R$, то для реакционного объема получим:

$$
V_{P}=4 \pi R^{2} \Delta R
$$

С учетом закона действующих масс химические потоки $J_{\text {п }}$ - прямой и $J_{\mathrm{O}}$ - обратной результирующей химической реакции, протекающей по схеме (1), имеют вид:

$$
\begin{array}{ll}
\frac{d N}{d t} \frac{1}{V_{\mathrm{p}}}=-k_{1} C_{1} C_{2} & J_{\Pi}=\frac{d N}{d t}=-V_{\mathrm{p}} k_{1} C_{1} C_{2} \\
\frac{d N}{d t} \frac{1}{V_{\mathrm{p}}}=-k_{2} C_{3}^{2} & J_{\mathrm{O}}=\frac{d N}{d t}=-V_{\mathrm{p}} k_{2} C_{3}^{2} .
\end{array}
$$

Учитывая выражение (5), полученное для реакционного объема, в последнем выражении (6) для потоков прямой и обратной реакции с учетом закона действующих масс получим:

$$
J_{\text {ПГ }}=-4 \pi R^{2} \Delta R k_{1} C_{1} C_{2} ; J_{0}=-4 \pi R^{2} \Delta R k_{2} C_{3}^{2} .
$$

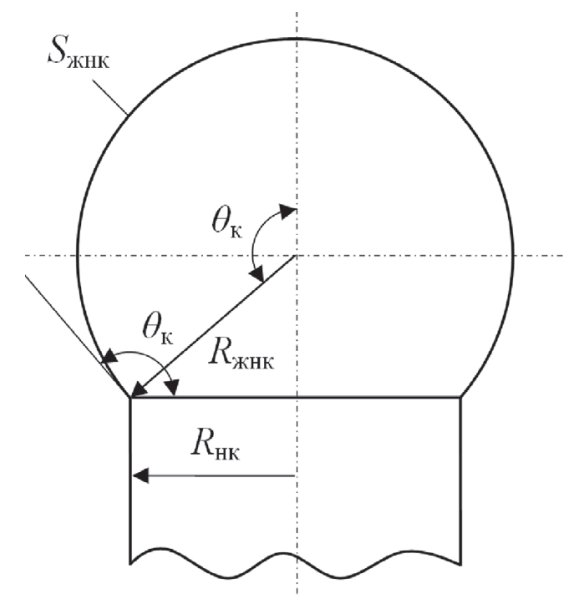

Рис. 2. Переход от потока, приводящего к росту кристалла, к его скорости роста

[Fig. 2. The transition from the flow leading to the growth of the crystal, to its growth rate]
Возможность протекания обратной гетерогенной химической реакции взаимодействия кремния с хлористым водородом (1) на поверхности жидкого сплава, содержащего обычно два компонента, определяется вероятностью одновременного соударения молекул $\mathrm{HCl}$ из газовой фазы с одним атомом кремния расплава. Эта вероятность пропорциональна поверхностной концентрации атомов кремния в жидком сплаве на вершине HK, которую обозначим $C_{s}$. Учтем мольную концентрацию атомов кремния в расплаве на вершине НК в выражении (7), которая определяет величину потока обратной химической реакции травления кремния $J_{0}$ :

$$
J_{\mathrm{O}}=-4 \pi R^{2} \Delta R C_{\mathrm{s}} k_{2} C_{3}^{2} .
$$

Кроме уже выше введенных диффузионного потока и химических потоков прямой о обратной гетерогенной реакции имеется атомарный поток кремния, возникающий в результате испарения с поверхности жидкого сплава на вершине НК. Величина атомарного потока испарения зависит от давления насыщенного пара испаряющегося вещества и обычно не велика. Однако для достаточно малых объектов давление насыщенного пара становится значительным и растет по мере уменьшения их размера по экспоненциальному закону.

Для наноразмерных НК размерный эффект становится существенным и его необходимо учитывать наряду с диффузионными и химическими потоками.

Учтем поток физического испарения с поверхности жидкости на вершине НК. Для этого определим плотность потока атомов насыщенного пара при физическом испарении. В стационарном случае для простого однокомпонентного вещества с плоской твердой или жидкой поверхностью поток испарения равен потоку конденсации. Тогда плотность потока атомов испаряющегося вещества $j_{\text {н }}$ пропорциональна числу соударений испаряющихся атомов пара с единицей поверхности жидкости или кристалла за единицу времени:

$$
j_{\text {н }}=\frac{1}{4} C_{\text {нг }} \sqrt{\frac{8 k_{\mathrm{E}} T}{\pi m}} .
$$

Здесь $C_{\text {нп }}$ - концентрация частиц насыщенного пара испаряющегося вещества (количество частиц пара на единицу объема), $k_{\text {Б }}$ - постоянная Больцмана, $T$ - температура, $m$ - масса атома (молекулы) пара.

Пусть поверхность жидкого сплава на вершине НK - сфера с радиусом $R$, как показано на рис. 1 , тогда для потока атомов испаряющегося вещества $J_{\text {н }}$ можно записать: 


$$
J_{\text {н }}=\pi R^{2} C_{s} C_{\text {нп }} \sqrt{\frac{8 k_{\mathrm{E}} T}{\pi m}},
$$

Здесь $C_{\mathrm{s}}$ - мольная доля атомов кремния на поверхности жидкого сплава на вершине НК. Так как НК растут по механизму пар-жидкость-кристалл, то в расплаве присутствуют два компонента - это кристаллизующееся вещество и вещество, образующее расплав. Будем считать поток испарения атомов кристаллизующегося вещества с поверхности расплава $J_{\text {н }}$ пропорциональным поверхностной концентрации $C_{\mathrm{s}}(2)$, и для плоской поверхности концентрацию насыщенного пара над расплавом запишем в виде: $C_{0 \mathrm{H}}=C_{\mathrm{s}} C_{\mathrm{H \Pi}}$.

Для НК малых радиусов существенна зависимость давления насыщенного пара от кривизны поверхности жидкой фазы. Если жидкая фаза представляет собой сплав, то в выражении для размерного эффекта следует учитывать атомную концентрацию испаряющегося вещества в сплаве:

$$
C_{\text {н }}=C_{s} C_{\text {Он }} \exp \left(\frac{2 \Omega \alpha_{\text {жг }}}{R_{\text {жнк }} k_{\mathrm{E}} T}\right),
$$

где $C_{\mathrm{s}}$ - относительная атомная концентрация испаряющегося вещества в сплаве, $C_{\text {н }}-$ концентрация насыщенного пара над сферической поверхностью жидкой фазы с радиусом кривизны $R_{\text {жнк }}, C_{\text {он }}-$ концентрация насыщенного пара над плоской поверхностью, $\alpha_{\text {жг }}$ - поверхностная энергия жидкого сплава на границе жидкость-газ.

Учтем (11) в выражении (10):

$$
J_{\text {н }}=\pi R^{2} C_{\mathrm{s}} C_{\text {он }} \sqrt{\frac{8 k_{\mathrm{Б}} T}{\pi m}} \exp \left(\frac{2 \Omega \alpha_{\text {жг }}}{R_{\text {жнк }} k_{\mathrm{B}} T}\right) .
$$

Выражение (12) - это испаряющийся поток атомов кристаллизующегося вещества, обеспечивающий оболочку насыщенного пара над поверхностью жидкости в слое $\Delta R$ (рис. 1).

Испаряющийся атомарный поток кремния вступает в реакцию:

$$
\mathrm{Si}_{(\text {ar })}+2 \mathrm{HCl}_{(\Gamma)} \rightarrow \mathrm{SiCl}_{2(\Gamma)}+\mathrm{H}_{2(\Gamma)}
$$

Как следует из реакции (13) один атом кремния взаимодействует с двумя молекулами хлористого водорода, образуя по одной молекуле дихлорида кремния и водорода. В виду высокой реакционной способности атомарного кремния будем считать, что реакция (13) не обратима.

Учтем соответствующие потоки, возникающие в результате испарения атомов кремния с поверхности расплава (4) по схеме реакции (1), в соответствующих выражениях, определяющих диффузионные потоки (2):

$$
\begin{aligned}
& J_{1}=4 \pi D_{1} R\left(C_{01}-C_{1}\right)+\pi R^{2} C_{s} C_{\text {0н }} \sqrt{\frac{8 k_{\mathrm{E}} T}{\pi m}} \exp \left(\frac{2 \Omega \alpha_{\text {жг }}}{R_{\text {жнк }} k_{\mathrm{b}} T}\right) ; \\
& J_{2}=4 \pi D_{2} R\left(C_{02}-C_{2}\right)+\pi R^{2} C_{s} C_{0 \mathrm{H}} \sqrt{\frac{8 k_{\mathrm{E}} T}{\pi m}} \exp \left(\frac{2 \Omega \alpha_{\text {жг }}}{R_{\text {жнк }} k_{\mathrm{B}} T}\right) ; \\
& J_{3}=4 \pi D_{3} R\left(C_{3}-C_{03}\right)-2 \pi R^{2} C_{s} C_{0 \mathrm{H}} \sqrt{\frac{8 k_{\mathrm{E}} T}{\pi m}} \exp \left(\frac{2 \Omega \alpha_{\text {жг }}}{R_{\text {жнк }} k_{\mathrm{b}} T}\right) .
\end{aligned}
$$

Учитывая соотношения, которые следуют из выражений, приведенных выше (2), (3), (4):

$$
C_{1}=C_{01}-\Delta C_{1} ; C_{2}=C_{02}-\Delta C_{2} ; C_{3}=C_{03}+\Delta C_{3},
$$

и воспользовавшись выше приведенным выражением (4), запишем:

$$
\begin{aligned}
& C_{1}=C_{01}-\Delta C_{1} ; \\
& C_{2}=C_{02}-\Delta C_{1} \frac{D_{1}}{D_{2}} ; \\
& C_{3}=C_{03}+2 \Delta C_{1} \frac{D_{1}}{D_{3}} .
\end{aligned}
$$

Учитывая выражение $(15)$ в $(7,8)$ для химических потоков прямой реакции выделения кремния и обратной реакции его травления, получим:

$$
\begin{gathered}
J_{\Pi}=-4 \pi R^{2} \Delta R k_{1}\left(C_{01}-\Delta C_{1}\right)\left(C_{02}-\Delta C_{1} \frac{D_{1}}{D_{2}}\right) ; \\
J_{0}=-4 \pi R^{2} \Delta R C_{s} k_{2}\left(C_{03}+\Delta C_{1} 2 \frac{D_{1}}{D_{3}}\right)^{2} .
\end{gathered}
$$

Поток атомов кремния - $J_{\mathrm{p}}$ или ростовой поток, приводящий к росту уса и выделяющийся за счет химической реакции на поверхности расплава на вершине НК, запишем как разность потоков прямой (выделения кремния) и обратной гетерогенной химической реакции (травления кремния) в соответствии со стехиометрией схемы реакции (1). В данном выражении учтем поток физического испарения атомарного кристаллизующегося вещества с поверхности жидкой фазы двухкомпонентного расплава:

$$
\begin{aligned}
J_{\mathrm{P}}= & 4 \pi R^{2} \Delta R\left[k_{1}\left(C_{01}-\Delta C_{1}\right)\left(C_{02}-\Delta C_{1} \frac{D_{1}}{D_{2}}\right)-\right. \\
& \left.-C_{\mathrm{s}} k_{2}\left(C_{03}+\Delta C_{1} 2 \frac{D_{1}}{D_{3}}\right)^{2}\right]- \\
& -\pi R^{2} C_{\mathrm{s}} C_{\text {0н }} \sqrt{\frac{8 k_{\mathrm{b}} T}{\pi m}} \exp \left(\frac{2 \Omega \alpha_{\text {жг }}}{R_{\text {жнк }} k_{\mathrm{E}} T}\right) \cdot
\end{aligned}
$$

Учитывая стехиометрию схемы химической реакции (1), приводящей к росту уса, запи- 
шем связи между потоками роста и диффузии в виде:

$$
J_{\mathrm{P}}=J_{1}=J_{2}=\frac{1}{2} J_{3} .
$$

Соотношение $J_{\mathrm{p}}=J_{1}$, с учетом (10), (2) примет вид:

$$
\begin{aligned}
& 4 R^{2} \Delta R\left[k_{1}\left(C_{01}-\Delta C_{1}\right)\left(C_{02}-\Delta C_{1} \frac{D_{1}}{D_{2}}\right)-\right. \\
& \left.-C_{\mathrm{s}} k_{2}\left(C_{03}+\Delta C_{1} 2 \frac{D_{1}}{D_{3}}\right)^{2}\right]- \\
& -R^{2} C_{s} C_{\text {Он }} \sqrt{\frac{8 k_{\mathrm{Б}} T}{\pi m}} \exp \left(\frac{2 \Omega \alpha_{\text {жг }}}{R_{\text {жнк }} k_{\mathrm{Б}} T}\right)= \\
& =4 D_{1} R \Delta C_{1}+R^{2} C_{s} C_{\text {0н }} \sqrt{\frac{8 k_{\mathrm{E}} T}{\pi m}} \exp \left(\frac{2 \Omega \alpha_{\text {жг }}}{R_{\text {жнк }} k_{\mathrm{E}} T}\right) . \\
& a=\left(k_{1} \frac{D_{1}}{D_{2}}-4 C_{\mathrm{s}} k_{2} \frac{D_{1}^{2}}{D_{3}^{2}}\right) ; \\
& b=\frac{D_{1}}{R \Delta R}+d \text {; } \\
& d=4 C_{\mathrm{s}} k_{2} C_{03} \frac{D_{1}}{D_{3}}+k_{1}\left(C_{01} \frac{D_{1}}{D_{2}}+C_{02}\right) \text {; } \\
& c=k_{1} C_{01} C_{02}-k_{2} C_{\mathrm{s}} C_{03}^{2}- \\
& -\frac{C_{s} C_{0 \mathrm{H}}}{2 \Delta R} \sqrt{\frac{8 k_{\mathrm{E}} T}{\pi m}} \exp \left(\frac{2 \Omega \alpha_{\text {жг }}}{R_{\text {жнк }} k_{\mathrm{E}} T}\right) .
\end{aligned}
$$

Решение уравнения (19) с учетом (20) запишется:

$$
\Delta C_{1(1,2)}=\frac{\left(\frac{D_{1}}{R \Delta R}+d\right) \pm \sqrt{\left(\frac{D_{1}}{R \Delta R}+d\right)^{2}-4 a c}}{2 a} .
$$

С учетом (18) $J \mathrm{p}=\frac{1}{2} J_{3}$ выражение для потока, приводящего к росту НК примет вид:

$$
J_{\mathrm{P}(1,2)}=\frac{4 \pi D_{1} R}{a}\left[\left(\frac{D_{1}}{R \Delta R}+d\right) \pm \sqrt{\left(\frac{D_{1}}{R \Delta R}+d\right)^{2}-4 a c}\right] .
$$

Перейдем от последнего выражения (22) для потока, приводящего к росту $\mathrm{HK} J_{\mathrm{P}(1,2)}$, к скорости роста уса $V_{\text {Hк(1,2) }}$.

Так как площадь сферической поверхности шаровой жидкой фазы равна площади поверхности шарового слоя расплава на вершине уса $S_{\text {жнк }}$ (рис. 2), то площадь плоского фронта кристаллизации уса определится в виде:

$$
\pi R_{\mathrm{HK}}^{2}=2 \pi R^{2}\left(1+\operatorname{Cos} \theta_{\mathrm{K}}\right) .
$$

Введем обозначение $\alpha=1+\operatorname{Cos} \theta_{\text {к. }}$.

Из (23) следует, что связь радиуса уса $R_{\text {нк }}$ с радиусом сферы шаровой жидкой фазы имеет вид:

$$
R=\frac{R_{\mathrm{HK}}}{\sqrt{2 \alpha}}
$$

Связь потока атомов кремния (ростового потока) $J_{\text {p }}$ со скоростью роста уса можно записать в виде:

$$
\pi R_{\mathrm{HK}}^{2} V_{\mathrm{HK}(1,2)}=J_{\mathrm{P}(1,2)} \Omega,
$$

где $V_{\text {нк(1.2) }}$ - скорость, с которой растет кристалл, $\Omega$ - объем атома кремния.

Тогда скорость роста уса, с учетом выражения (22), может быть записана в следующем виде:

$$
\begin{gathered}
V_{\mathrm{HK}(1,2)}=\frac{2 \Omega D_{1}}{a R_{\mathrm{HK}} \sqrt{2 \alpha}}\left[\left(\frac{\sqrt{2 \alpha} D_{1}}{R_{\mathrm{HK}} \Delta R}+d\right) \pm\right. \\
\pm \sqrt{\left.\left(\frac{\sqrt{2 \alpha} D_{1}}{R_{\mathrm{HK}} \Delta R}+d\right)^{2}-4 a c\right] .}
\end{gathered}
$$

На рис. 3 показана зависимость скорости роста НК от его радиуса (26).

Вариант, соответствующий кривой 1 на рис. 3 на практике не реализуется. Рост НК всегда ограничен по радиусу, то есть радиус кристалла не может превышать некоторого максимального значения. Максимальное значение радиуса НК определяется условиями роста и обычно не превышает $200 \mu \mathrm{m}$.

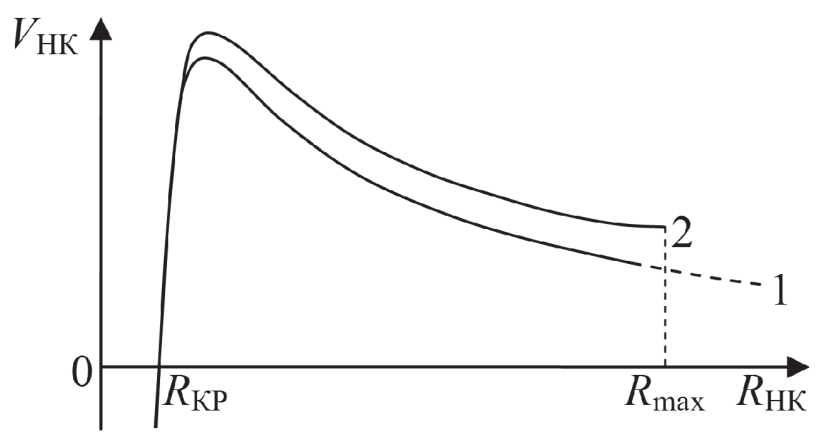

Рис. 3. Зависимость скорости роста НК $V_{\text {нК }}$ от его радиуса $R_{\text {нк. }}$ Кривая 1 - скорость роста НК стре-

мится к нулю при его радиусе, стремящемся к бесконечности; кривая 2 - НК с радиусами, превышающими $R_{\max }$, расти не могут

[Fig. 3. Dependence of the growth rate of NK $V_{\mathrm{NA}}$ on its radius $R_{\mathrm{NA}}$. Curve $1-\mathrm{NC}$ growth rate tends to zero with its radius tending to infinity; curve $2-$

$\mathrm{NC}$ with radii exceeding $R_{\max }$ cannot grow] 
Рассмотрим подкоренное выражение. Из условия равенства нулю покоренного выражения (26) найдем максимальный радиус HK $R_{\max }$ :

$$
R_{\max }=\frac{\sqrt{2 \alpha} D_{1}}{2 \Delta R(\sqrt{a c}-d)} .
$$

Радиус НК $R_{\mathrm{KP}}$, при котором скорость роста обращается в нуль, найдем из условия равенства нулю слагаемого 4ac в подкоренном выражении, которое сводится к виду:

$$
\begin{gathered}
k_{1} C_{01} C_{02}-k_{2} C_{\mathrm{s}} C_{03}^{2}= \\
=\frac{C_{s} C_{0 \mathrm{H}}}{2 \Delta R} \sqrt{\frac{8 k_{\mathrm{5}} T}{\pi m}} \exp \left(\frac{2 \Omega \alpha_{\text {жг }}}{R_{\text {жнк }} k_{\mathrm{5}} T}\right) .
\end{gathered}
$$

Откуда для $R_{\mathrm{KP}}$ с учетом геометрии на рис. 2 получим:

$$
R_{\mathrm{KP}}=\frac{2 \Omega \alpha_{\text {жг }} \sin \theta_{\mathrm{K}}}{k_{\mathrm{L}} T \ln \left[\frac{2 \Delta R\left(k_{1} C_{01} C_{02}-k_{2} C_{\mathrm{s}} C_{03}^{2}\right)}{C_{s} C_{0 \mathrm{H}} \sqrt{\frac{8 k_{\mathrm{b}} T}{\pi m}}}\right]}
$$

Как следует из выражения (29), величина радиуса $Н \mathrm{~K} R_{\mathrm{KP},}$ при котором скорость его роста становится нулевой, зависит от исходного состава газовой смеси, констант скоростей схемы химической реакции, свойств расплава на вершине уса и температуры, при которой происходит его рост.

\section{ЗАКЛЮЧЕНИЕ}

В работе рассмотрена модель роста НК на примере усов кремния для открытой проточной системы $\mathrm{H}_{2}+\mathrm{SiCl}_{4}$ атмосферного давления в случае, когда процесс роста уса контролируется гетерогенной химической реакцией выделения атомарного кремния на поверхности расплава на границе жидкость-газ. В модели учтен поток испарения кристаллизующегося вещества с поверхности жидкой фазы и зависимость давления насыщенного пара от поперечного размера кристалла.

Получена зависимость скорости роста НК от их радиуса и технологических параметров процесса роста (скорость потока газовой смеси в реакторе, температура (26), состав газовой смеси [14]), которая имеет максимум и ограничена в области больших радиусов кристаллов. При достаточно малых радиусах НК скорость роста обращается в нуль.

Получены выражения, определяющие максимальный радиус НК, при котором рост прекращается, и минимальный радиус, при котором скорость роста НК обращается в нуль.
Модель роста НК, контролируемого гетерогенной химической реакцией, с учетом размерного эффекта дала практические результаты, объясняющие известные экспериментальные данные. Результаты моделирования могут быть использованы для роста НК различных веществ в условиях контроля процесса гетерогенной химической реакцией выделения кристаллизующегося вещества. Результаты работы могут быть использованы для управления и оптимизации процесса роста НК.

\section{КОНФЛИКТ ИНТЕРЕСОВ}

Авторы декларируют отсутствие явных и потенциальных конфликтов интересов, связанных с публикацией настоящей статьи.

\section{СПИСОК ЛИТЕРАТУРЫ}

1. Вагнер Р. Монокристальные волокна и армированные ими материалы / Под ред. А. Т. Туманова. М.: Мир, 1973, 64 с.

2. Гиваргизов Е.И. Рост нитевидных и пластинчатых кристаллов из пара. М.: Наука, 1977, 304 с.

3. Небольсин А. А., Щетинин А. А. Рост нитевидных кристаллов. Воронеж: ВГТУ, 2003, 620 с.

4. Дубровский В. Г., Цырлин Г. Э., Устинов В. М. Полупроводниковые нитевидные нанокристаллы: синтез, свойства, применения // ФТП, 2009, т. 43(12), с. $1585-1628$.

5. Антипов С. А., Дрожжин А. И., Рощупкин А. М. Релаксационные явления в нитевидных кристаллах полупроводников. Воронеж: ВГУ, 1987, 192 с.

6. Дрожжин А. И. Преобразователи на нитевидных кристаллах P-Si<111>. Воронеж: ВГПИ, 1984, 241 с.

7. Spinelli P., Verschuuren M. A., Polman A. Broadband omnidirectional antireflection coating based on subwavelength surface Mie resonators // Nat. Commun., 2012,v.3(1), p.692.DOI: https://doi.org/10.1038/ ncomms 1691

8. Zhang R., Zhang Y., Zhang Q., Xie H., Qian W., Wei F. Growth of half-meter long carbon nanotubes based on schulz-flory distribution // ACS Nano, 2013, v. 7 (7), pp. 6156-6161. DOI: https://doi.org/10.1021/ $\mathrm{nn} 401995 \mathrm{z}$

9. Козенков О. Д. Модель роста нитевидного кристалла, лимитируемого гетерогенной химической реакцией // Неорганические материалы, 2014, т. 50 (11), с. 1238. DOI: https://doi.org/10.7868/ S0002337X14110104

10. Козенков О. Д. Зависимость скорости роста нитевидного кристалла, лимитируемого гетерогенной химической реакцией, от состава жидкой фазы // Конденсированные среды и межфазные границы, 2016. т. 18(3), с. 338-344. Режим доступа: https://journals.vsu.ru/kcmf/article/view/141/99 (дата обращения: 02.12.2019) 
11. Shchetinin A. A., Bubnov L. I., Kozenkov O. D., Tatarenkov A. F. Influence of various impurities on the axial growth rate of silicon whiskers. Известия Академии наук СССР. Неорганические материалы, 1987, т. 23(10), с. 1589-1592.

12. Козенков О. Д., Горбунов В. В. Модель теплового баланса бесконечно длинного нитевидного кристалла // Неорганические материалы, 2015, т. 51(5), c. 576-580. DOI: https://doi.org/10.7868/ S0002337X15050073

13. Козенков О.Д., Щетинин А.А., Горбунов В. В., Сычев И. В. Зависимость скорости роста нитевидного кристалла, лимитируемого гетерогенной химической реакцией, от состава газовой фазы при больших концентрациях тетрахлорида кремния // Вестник ВГТУ, 2016, т. 13(4), с. 78-184.

14. Козенков О. Д., Косырева Л. Г. Зависимость скорости роста нитевидного кристалла, лимитируемого гетерогенной химической реакцией, от состава газовой фазы // Неорганические материалы, 2015, т. 51(11), с. 1255-1259. DOI: https://doi. org/10.7868/S0002337X15100097
15. Даринский Б. М., Козенков О. Д., Щетинин А. А. О зависимости скорости роста нитевидных кристаллов от их диаметра // Известия вузов. Физика, 1986, т. 32(12), с. 18-22.

16. Щетинин А. А., Козенков О. Д., Небольсин В. А. О зонах питания нитевидных кристаллов кремния растущих из газовой фазы // Известия вузов, Физика, 1989, т. 32(6), с. 115-116.

17. Щетинин А. А., Дунаев А. И., Козенков О. Д О травлении монокристаллов кремния через жидкую фазу и образовании систем обычных и «отрицательных» нитевидных кристаллов. Воронеж: ВГПИ, 1981, $9 \mathrm{c}$.

18. Козенков О. Д., Козьяков А. Б., Щетинин А. А. О конусности нитевидных кристаллов кремния // Известия вузов, Физика, 1986, т. 29(9), с. 115-117.

19. Козенков О. Д. Конусность нитевидного кристалла, обусловленная гетерогенной химической реакцией // Неорганические материалы, 2016, т. 52(3), c. 279-284. DOI: $110.7868 /$ S0002337X16030064

\title{
Growth Model of Whisker Controlled by Heterogeneous Chemical Reaction Considering the Size Effect
}

\author{
(c) 2019 O. D. Kozenkov ${ }^{1 \bowtie}$, D. A. Zhukalin² ${ }^{2}$ I. O. Baklanov ${ }^{1}$, L. G. Cosycreva ${ }^{1}$, \\ A. Y. Klimov ${ }^{1}$, I. S. Obozny ${ }^{1}$ \\ ${ }^{1}$ Military Educational and Scientific Center of the Air Force \\ «N.E. Zhukovsky and Y.A. Gagarin Air Force Academy» (Voronezh) \\ 54 A, Bol'shevikov str., 394064 Voronezh, Russian Federation \\ Voronezh State University \\ 1, Universitetskaya pl., 394018 Voronezh, Russian Federation
}

\begin{abstract}
Purpose. The subject of this study were whisker, which are quasi-one-dimensional single-crystal objects with a high degree of sophistication and strength close to the theoretical model. The transverse dimensions of whisker can be from hundreds of micrometres to tens of nanometres, and their length is several orders of magnitude higher than the diameter. The study is devoted to the investigation of the kinetics of filamentary crystal growth controlled by a heterogeneous chemical reaction of crystallizing substance release. The goal of the study was the establishment of kinetic laws of growth of whisker with transverse dimensions of less than $1 \mu \mathrm{m}$, when the size effect associated with an increase in the pressure of saturated vapour over a solid or liquid surface with an increase in its curvature becomes significant.

Methods and methodology. Physical and computer modelling methods were used in the study. Silicon whisker were obtained in an open flow system $\mathrm{SiCl}_{4}+\mathrm{H}_{2}$. Metal particles $(\mathrm{Cu}, \mathrm{Au}, \mathrm{Pt}, \mathrm{Ni}$, $\mathrm{Ag}, \mathrm{Sn}$ ) were deposited on the prepared single-crystal $\mathrm{Si}<111\rangle$ substrates, which initiated crystal growth by the vapour-liquid-crystal mechanism. Silicon substrates with metal particles were placed in a horizontal reactor located in a furnace with radiation heating.
\end{abstract}

Oleg D. Kozenkov, e-mail: kozenkov_w@mail.ru 
Results. The earlier proposed model of the growth of silicon whisker, controlled by a chemical reaction at the liquid-gas interface, was supplemented by taking into account the pressure of saturated silicon vapour in the melt at the top of the crystal. For crystals of sufficiently small sizes, the saturated vapour pressure of the crystallizing substance becomes so significant that crystal growth ceases. The model involves diffusion delivery of the starting substances, and the removal of reaction products into a thin surface gas layer at the liquid-gas interface, where the concentrations of the reagents remain constant. The concentration of reagents in this layer determines the rate of the chemical reaction of the precipitation of the crystallizing substance. The flow arising due to the pressure of saturated silicon vapour over the melt was taken into account, providing that the evaporating atoms almost completely interact with the reactants in the gas phase. The balance of diffusion, chemical and evaporation flows makes it possible to determine the growth rate of a filamentary crystal depending on its radius and technological parameters of the process. The dependence of the growth rate of a filamentary crystal on its radius has a maximum value and for sufficiently small transverse dimensions of the crystal it becomes zero. With sufficiently large radii, crystal growth ceases. The expression determining the maximum radius of the crystal at which growth becomes impossible was obtained.

Conclusions. In the growth model of a filamentary crystal controlled by a heterogeneous chemical reaction of crystallizing, the evaporation flow of the crystallizing substance from the surface of the liquid phase and the dependence of the saturated vapour pressure on the transverse size of the crystal were taken into account.

The dependence of the growth rate of nanocrystals on their radius and technological parameters of the growth process was obtained, which has a maximum and is limited in the region of large crystal radii. For sufficiently small radii of nanocrystals, the growth rate becomes zero.

Expressions determining the maximum radius of nanocrystals at which growth ceases, and the minimum radius at which the growth rate of nanocrystals becomes zero were obtained.

A growth model of nanocrystals controlled by a heterogeneous chemical reaction, taking into account the size effect, yielded practical results explaining the known experimental data. The simulation results can be used for the growth of nanocrystals of various substances under the control of the heterogeneous chemical reaction of precipitation of crystallizing substance and can be used for the control of the growth and optimization of the growth of nanocrystals.

Keywords: whisker heterogeneous chemical reaction, diffusion flow, chemical reaction flow, evaporation flow, minimum radius, maximum radius.

\section{CONFLICT OF INTEREST}

The authors declare the absence of obvious and potential conflicts of interest related to the publication of this article.

\section{REFERENCES}

1. Vagner R. Monokristal'nyye volokna i armirovannyye imi materialy [Monocrystal fibers and materials reinforced by them]/Ed. by: A. T. Tumanova. Moscow, Mir Publ., 1973. 464 p. (in Russ.)

2. Givargizov Ye. I. Rost nitevidnykh i plastinchatykh kristallov iz para [The growth of whiskers and lamellar crystals of steam]. Moscow, Nauka Publ., 1977, 304 p. (in Russ.)

3. Nebol'sin A. A., Schetinin A. A. Rost nitevidnykh kristallov [Growth of whiskers]. Voronezh: VSTU Publ., 2003, 620 p. (in Russ.)

4. Dubrovskii V. G., Cirlin G. E., Ustinov V. M. Semiconductor nanowhiskers: Synthesis, properties, and applications. Semiconductors, 2009, v. 43(12), pp. 15391584 . DOI: https://doi.org/10.1134/ S106378260912001X
5. Antipov S. A., Drozhzhin A. I., Roshchupkin A. M. Relaksatsionnyye yavleniya $v$ nitevidnykh kristallakh poluprovodnikov [Relaxation phenomena in semiconductor whiskers. Voronezh: Voronezh State University Publ., 1987, 192 p. (in Russ.)

6. Drozhzhin A. I. Preobrazovateli na nitevidnykh kristallakh $\mathrm{R}-\mathrm{Si}<111>$ [Converters on $\mathrm{P}-\mathrm{Si}$ whiskers $<111>$.]. Voronezh: VGPI Publ., 1984, 241 p. (in Russ.)

7. Spinelli P., Verschuuren M. A., Polman A. Broadband omnidirectional antireflection coating based on subwavelength surface Mie resonators. Nat. Commun., 2012, v. 3(1), p. 692. DOI: https://doi.org/10.1038/ ncomms 1691

8. Zhang R., Zhang Y., Zhang Q., Xie H., Qian W., Wei F. Growth of half-meter long carbon nanotubes based on schulz-flory distribution. ACS Nano, 2013, v. 7 (7), pp. 6156-6161. DOI: https://doi.org/10.1021/ nn401995z

9. Kozenkov O. D. A model for whisker growth limited by a heterogeneous chemical reaction. Inorganic Materials, 2014, v. 50(11), pp. 1146-1150. DOI: https://doi.org/10.1134/S0020168514110107 
10. Kozenkov O. D. Effect of liquid phase composition on the whisker growth rate limited by a heterogeneous chemical reaction Kondensirovannye sredy $i$ mezhfaznye granitsy [Condensed Matter and Interphases], 2016, v. 18(3), pp. 338-344. Available at: https://journals.vsu.ru/kcmf/article/view/141/99 (accessed 02.12.2019). (in Russ., abstract in Eng.)

11. Shchetinin A. A., Bubnov L. I., Kozenkov O. D., Tatarenkov A. F. Influence of various impurities on the axial growth rate of silicon whiskers. Izvestiya Akademii nauk SSSR. Neorganicheskiye materialy, 1987, v. 23(10), pp. 1589-1592. (in Ing.)

12. Kozenkov O. D., Gorbunov V. V. A model for the heat balance of an infinitely long whisker. Inorganic Materials, 2015, v. 51(5), pp.520-524. DOI: https://doi. org/10.7868/S0002337X15050073

13. Kozenkov O. D., Shetinin A. A., Gorbunov V. V., Sychev I. V. Dependence of the rate of whisker growth, limited type of heterogeneous chemical reactions, the composition of the gas phase at a greater concentration of silicon tetrachloride. Vestnik Voronezhskogo gosudarstvennogo tekhnicheskogo universiteta [Preceding of Voronezh State Technical University], 2016, v. 13(4), pp. 78-84. ). (in Russ., abstract in Eng.)

14. Kozenkov O. D., Kosyreva L. G. Effect of vaporphase composition on the whisker growth rate limited by a heterogeneous chemical reaction. Inorganic $M a-$ terials, 2015, v. 51(11), pp.1163-1167. DOI: https://doi. org/10.1134/S002016851510009X
15. Darinskiy B. M., Kozenkov O. D., Shchetinin A. A. O zavisimosti skorosti rosta nitevidnykh kristallov ot ikh diametra [On the dependence of the growth rate of whiskers on their diameter]. Izvestiya vuzov, Fizika [News of Universities, Physics], 1986, v. 32(12), pp. 18-22. (in Russ.)

16. Shchetinin A. A., Kozenkov O. D., Nebol'sin V. A. O zonakh pitaniya nitevidnykh kristallov kremniya rastushchikh iz gazovoy fazy [On the nutrition zones of silicon whiskers growing from the gas phase]. Izvestiya vuzov, Fizika [News of Universities, Physics], 1989, v. 32(6), pp. 115-116. (in Russ.)

17. Shchetinin A. A., Dunayev A. I., Kozenkov O. D O travlenii monokristallov kremniya cherez zhidkuyu fazu i obrazovanii sistem obychnykh $i$ «otritsatel'nykh» nitevidnykh kristallov [On the etching of silicon single crystals through the liquid phase and the formation of systems of ordinary and "negative" whiskers]. Voronezh. VGPI Publ., 1981, 9 p. (in Russ.)

18. Kozenkov O. D., Koz'yakov A. B., Shchetinin A. A. O konusnosti nitevidnykh kristallov kremniya [On the taper of silicon whiskers]. Izvestiya vuzov, Fizika [News of Universities, Physics], 1986, v. 29(9), pp. 115-117. (in Russ.)

19. Kozenkov O. D. Whisker taper induced by heterogeneous chemical reaction. Inorganic Materials, 2016, v. 52(3), pp. 239-243. DOI: https://doi. org/10.1134/S0020168516030067
Козенков Олег Дмитриевич - к. ф.-м. н, старший преподаватель кафедры физики, Военный учебно-научный центр ВВС «Военно-воздушная академия им. профессора Н. Е. Жуковского и Ю.А. Гагарина», Воронеж, Российская Федерация; e-mail: kozenkov_w@mail.ru. ORCID iD: https://orcid.org/0000-0003-2888-463X.

Жукалин Дмитрий Алексеевич - к. ф.-м. н, доцент кафедры физики полупроводников и микроэлектроники, Воронежский государственный университет, Воронеж, Российская Федерация; e-mail: d.zhukalin@mail.ru. ORCID iD: https:// orcid.org/0000-0002-0754-4989.

Бакланов Игорь Олегович - д. пед. н, доцент, заведующий кафедрой физики, Военный учебно-научный центр ВВС «Военно-воздушная академия им. профессора Н. Е. Жуковского и Ю.А. Гагарина», Воронеж, Российская Федерация; e-mail: baklanov_io@mail.ru. ORCID iD: https://orcid.org/0000-0001-7093-6048.
Oleg D. Kozenkov - Cand. Sci. (Phys.- Math.), Senior Lecturer of the Department of Physics, Military Educational and Scientific Center of the Air Force «N. E. Zhukovsky and Y. A. Gagarin Air Force Academy», Voronezh, Russian Federation; e-mail: kozenkov_w@mail.ru. ORCIDiD: https://orcid.org/00000003-2888-463X.

Dmitry Alekseevich Zhukalin - Cand. Sci. (Phys.Math.), Associate Professor of the Department of Physics of Semiconductors and Microelectronics, Voronezh State University, Voronezh, Russian Federation; e-mail: d.zhukalin@mail.ru. ORCID iD: https://orcid.org/0000-0002-0754-4989.

Igor O. Baklanov - Dr. Sci. (Ped.), Associate Professor, Head of the Department of Physics, Military Educational and Scientific Center of the Air Force «N.E. Zhukovsky and Y.A. Gagarin Air Force Academy», Voronezh, Russian Federation; e-mail: baklanov_io@mail.ru. ORCID iD: https://orcid.org/00000001-7093-6048. 
Косырева Людмила Геннадьевна - к. ф.-м. н, старший преподаватель кафедры физики, Военный учебно-научный центр ВВС «Военно-воздушная академия им. профессора Н. Е. Жуковского и Ю. А. Гагарина», Воронеж, Российская Федерация; e-mail: e-mail: lyudovikxiv@yandex.ru.ORCID iD: https://orcid.org/0000-0001-6157-1243.

Климов Александр Юрьевич - курсант, Военный учебно-научный центр ВВС «Военно-воздушная академия им. профессора Н. Е. Жуковского и Ю. А. Гагарина», Воронеж, Российская Федерация; ORCID iD: https://orcid.org/00000002-0424-2598.

Обозный Илья Сергеевич - курсант, Военный учебно-научный центр ВВС «Военно-воздушная академия им. профессора Н. Е. Жуковского и Ю.А. Гагарина», Воронеж, Российская Федерация; ORCID iD: https://orcid.org/0000-00019304-4632.
Lyudmila G. Cosycreva - Cand. Sci.(Phys.- Math.), Senior Lecturer of the Department of Physics, Military Educational and Scientific Center of the Air Force «N. E. Zhukovsky and Y. A. Gagarin Air Force Academy», Voronezh, Russian Federation; e-mail: lyudovikxiv@yandex.ru. ORCID iD: https://orcid. org/0000-0001-6157-1243.

Alexander Yu. Klimov - cadet, Military Educational and Scientific Center of the Air Force «N. E. Zhukovsky and Y. A. Gagarin Air Force Academy», Voronezh, Russian Federation; ORCID iD: https://orcid.org/0000-0002-0424-2598.

Ilya S. Obozny - cadet, Military Educational and Scientific Center of the Air Force «N. E. Zhukovsky and Y. A. Gagarin Air Force Academy», Voronezh, Russian Federation; ORCID iD: https://orcid.org/0000-0001-9304-4632. 